\title{
La violence politique au Congo-Kinshasa 1996-2006 : le décryptage d'un mode de participation politique revitalisé
}

\author{
Phidias Ahadi Senge Milemba ${ }^{1}$
}

\begin{abstract}
Résumé :- L'ambition de ce papier a consisté à remonter à la surface le fermant de la revitalisation de la violence dans le jeu politique congolais. Il démontre combien l'Etat congolais, laminé par des malaises sociaux, des contestations de légitimité et verrouillage des voies de lutte démocratique, a été un tremplin à l'explosion de la violence sous la bannière des mouvements politico-militaires AFDL, RCD, MLC, Mä̈-maï. Le tout dans l'objectif de désavouer les institutions politiques, et les substituer par un autre régime souhaité démocratique, dont la mission est d'établir un système social juste et équilibré, où la distribution des ressources collectives est fonction du mérite, de besoin et du statut des membres. Cependant, les attentes populaires quant à ce, sont restées déçues par le remplacement d'une dictature par une autre, dans un continuum d'oppressions aussi rédhibitoires que la crise démocratique des régimes répressifs précédents.
\end{abstract}

Mots clés : violence politique, participation politique, démocratie, malaise sociale, violence revitalisée.

Abstact :-This paper aims to point out the ferment of violence revitalization in the Congolese political game. The paper demonstrates how the Congolese state annihilated by social unrest, legitimacy contests, the locking of all the ways to democratic struggle, has been a springboard for the explosion of violence from AFDL, RCD, MLC, Mai-mai political and military movements. All these movements' goal has been to fight the political institutions and replace them by a desirable democratic regime whose mission will be the establishment of a fair, balanced and righteous social system where the members' merit, need and status will be taken into account in the distribution of collective resources. However people's expectations relative to the establishment of such a system have been disappointed since one form of dictatorship has been replaced by another with the continuity of repressions that are as appalling as the democratic crises of the proceeding repressive regimes.

Key words: political violence, political involvement, democracy, social unrest, revitalized violence.

\section{INTRODUCTION}

La réputation de la violence au Congo-Kinshasa a revêtu plusieurs lustres à travers des décennies : moyens d'insertion dans le jeu politique, expression du désespoir et des inégalités sociales, contestations du régime politique établi depuis la décolonisation sous le mot d'ordre de démocratie et/ou de bonne gouvernance, ... La violence est donc bien ce que martèle Philippe Braud, une forme de participation, elle-même parfois bien davantage: l'exigence d'un accès à l'exercice direct du pouvoir politique ${ }^{2}$. Cette forme de participation politique revitalisée au Congo-Kinshasa est, à n'en point douter, expliquée par le binôme caractéristique de l'Etat congolais 1996-2006 et bien au de-là de cette temporalité retenue pour l'analyse dans ces lignes.

D'une part, cette violence apparaîtrait utilitaire pour autant qu'elle a pu poser des germes d'une nouvelle dynamique sociétale à travers le dialogue inter-congolais tenu en 2002 en Afrique du Sud. Ces assises politiques avaient accouché d'un 'nouvel ordre politique national' qui avait permis le processus de la mise en œuvre d'une paix globale au Congo-Kinshasa, expression de la participation des élites rebelles aux institutions politiques consensuelles. L'euphorie autour de cet ouvrage combien ambitieux fut, cependant, de courte durée. La violence a demeuré banalisé et servir les individus ou les groupes qui veulent faire entendre leur voix, faire prévaloir leurs opinions ou même satisfaire leurs intérêts mesquins, leur soif du pouvoir et de domination.

Il s'agit, pour reprendre l'idée de Philippe Braud, des formes paradoxales de participation qui visent à contester les règles du jeu institutionnel normal en tentant d'y substituer d'autres formes ${ }^{3}$. D'autre part, cette

\footnotetext{
${ }^{1}$ Professeur Associé, Faculté des Sciences Sociales, Administratives et Politiques, Département des Sciences Politiques et Administratives, Université de Goma, République Démocratique du Congo. Ce papier, objet d'une réflexion aux Annales de l'Université de Goma,Volume V, Numéro 2, 2015, pp.25-34, est, aujourd'hui, revu et augmenté.

${ }^{2}$ P. Braud, Sociologie politique, Paris, L.G.D.J, 2011, p.424.

${ }^{3}$ P. Braud, Op. cit., p.424. 
violence apparaitrait on ne peut plus désastreuse tant qu'elle est restée rythmer la vie en société avec comme chef d'accusation: la désorganisation, les destructions, la déshumanisation de la société congolaise ayant compté des morts parmi les siens, par milliers puis par millions.

Le défis de l'ouverture du système politique congolais aux valeurs républicaines lancées aux titulaires des rôles politiques et au souverain primaire ainsi qu'à tous ceux qu'intéressent le devenir démocratique de ce pays, a demeuré, malencontreusement, stérile quant à ce. La revitalisation de l'insertion violente dans le jeu politique congolais, du reste un signe de danger pour le système politique, et, de l'avis de Philippe Braud, un aveu d'un échec ou d'un refus ${ }^{4}$, semble mieux indiqué si et seulement si elle est démocratisant et, à l'entendement de Fweley Diangitukwa, permet parfois la conquête du pouvoir, surtout dans les Etats du tiers monde où le pouvoir et l'autorité sont constamment contestés 5 .

Ce raisonnement donne à affirmer que la violence est une ressource politique comme les autres, un mode de participation politique dans une infinité des formes, dont se servent les gouvernés comme certains gouvernants pour influencer le fonctionnement du système politique ; contester le régime politique en place en tentant d'y substituer un autre, souhaité favorable au noyau dur de la démocratie, selon lequel, la souveraineté appartient à l'ensemble des citoyens qui l'exercent à l'occasion du vote libre, démocratique, transparent et régulier ou par une autre procédure institutionnelle (referendum, pétition), le pluralisme politique supposant le multipartisme, le libéralisme politique, ... Et pour le dire autrement, un régime favorable au développement de l'individu et de la société.

Cette note se préoccupe donc de fournir des éléments de réponse à la question fondamentale de savoir, pourquoi la violence est-elle devenue un mode de participation politique revitalisé au Congo-Kinshasa, où les moyens d'acquisition du pouvoir d'Etat sont plus normés et constitutionnellement garantis ? La réponse à cette question dépasse amplement le cadre de cette dissertation. En effet, un Etat laminé par des malaises sociaux, des contestations de légitimité et verrouillage des voies de lutte démocratique offrirait inévitablement la possibilité de recours à la violence pour désavouer les institutions politiques, la revitaliser pour substituer le régime contesté par un autre, démocratique et de nature à établir un système social juste et équilibré où la distribution/redistribution des ressources collectives est fonction du mérite, de besoin et du statut des membres, ingrédient aux flux des soutiens populaires au régime.

Cette réflexion a donc pour ambition de remonter à la surface le fermant de la revitalisation de la violence dans le jeu politique congolais. Pour ce faire, outre le contenu qu'elle donnera au concept de violence politique, elle s'articulera sur la violence politique sous l'Alliance des Forces Démocratiques pour la Libération du Zaïre (AFDL), le Rassemblement Congolais pour la Démocratie (RCD), le Mouvement de Libération du Congo (MLC) et sur la violence entreprise par les mouvements de résistance maï-maï et en tirer des leçons.

\section{QU'EST-CE QUE LA VIOLENCE POLITIQUE ?}

La violence est une notion complexe à cerner le contenu. Elle est au cœur de plusieurs perceptions souvent antithétiques. Cependant, il n'est nul besoin de s'ingérer dans ce débat querellé entre spécialistes. Tout de même, l'analyse de science politique se doit de proposer une acception qui transcende les particularismes d'approches au profit d'une autre, à la limite, unificatrice de ce qu'elles ont de commun.

De ce fait, la littérature spécialisée, wébérienne notamment, définit ce qu'il convient de nommer violence politique, une prérogative exclusive du pouvoir politique. Cette approche dont l'Etat détient le monopole de la violence légitime ou supposée légitime, reste évidemment limitée et peu satisfaisante au Congo-Kinshasa notamment, où cet imperium est dorénavant démystifié. L'Etat y a déjà perdu le monopole de violence qu'il partage désormais avec des individus (les seigneurs de guerre) et les groupes armées organisés qui contrôlent une partie de son territoire ainsi que son administration.

Pour un œil exercé, ce raisonnement donne à affirmer que la violence n'est pas l'apanage du seul pouvoir d'Etat dans les démocraties contemporaines; elle est aussi un mode d'expression sociale, contestataire par exemple. Le recours à une violence calculée et calculatrice est le fait d'organisations extérieures, mais aussi indigènes, donc c'est le mode "normal » d'insertion dans le jeu politique, compte tenu de leurs objectifs et moyens réels dont elles disposent pour maximiser leur affirmation politique ${ }^{6}$. De plus, elle exclut d'autres formes de violence (inter sociale, contestataire, révolutionnaire, etc.) qui peuvent devenir politique à la suite de la requalification des faits : la signification politique de l'acte.

En réalité, deux conditions permettent de caractériser le passage à la violence politique, présenté avec pertinence par Ted Gurr. Premièrement, le passage à la violence politique est favorisé par la diffusion des

\footnotetext{
${ }^{4}$ P. Braud,Idem, p.469.

${ }_{6}^{5}$ Fweley Diangitukwa, Qu'est-ce que le pouvoir? Paris, L'harmattan, 2004, p.62.

${ }^{6} \mathrm{P}$. Braud, «La violence : repères et problèmes», in Cultures\&Conflits, 09-10, printemps-été, [En ligne], mis en ligne le 13 mars 2006. URL http://conflits.revues.org/index406.html. Consulté le 4 septembre 2012. 
normes éthiques justificatives ${ }^{7}$. A cet égard, l'explosion de la violence sous la marque de l'AFDL était fondée sur une justification morale : lutte contre la corruption, l'accaparement de l'Etat, la partitocratie, le népotisme, etc. Il a s'agit donc de se faire une image du libérateur en justifiant son option pour la guerre contre le régime en place, qui représente un danger pour le peuple, dénoncer la responsabilité de ce régime contesté dans l'émergence des crises politiques, économiques, socioculturelles, en dressant un tableau beaucoup plus sombre d'indices intolérables de la situation, avec des accents affreux, pour, et enfin, se proposer comme une meilleure alternative par la formulation des accomplissements souhaitables du peuple. L'objectif reste celui de gagner la confiance populaire, de se façonner une opinion publique d'un pouvoir responsable, dynamique, entrepreneur, démocratique et attentif aux problèmes et attentes populaires. D'où, affirme Philippe Braud, peuvent être plus ou moins répandues des convictions selon lesquelles la résistance à l'oppression est un devoir qui implique éventuellement l'emploi de la violence ${ }^{8}$.

En effet, cette violence agit de telle manière que son centre de gravité reste les secteurs les plus sensibles de la société : répartition des revenus nationaux, la gouvernance participative, le contrôle citoyen, etc. Il faut cependant reconnaître que la violence n'est pas un acte homogène, et qu'au contraire, mille et une expressions exigent de considérer chaque forme comme un cas d'école : de fait, il n'est pas pertinent de loger à la même enseigne toutes les formes de la violence. Toutefois, le trait commun à toute violence, même de basse intensité, est d'humilier les victimes, de leur faire éprouver leur fragilité, voir leur impuissance momentanée?

Deuxièmement, le passage à la violence est favorisé par la conviction selon laquelle elle peut se révéler payante ${ }^{10}$. Si en effet, les élites des mouvements insurrectionnels RCD, MLC etc. ont été des nouveaux maîtres des institutions politiques de la transition 2003-2006 d'après le "contrat de paix" du 17 décembre 2002, le risque encouru est resté d'encourager et de pousser les autres acteurs institutionnels et/ou non étatiques à mobiliser, à leur tour, cette ressource politique qui s'est révélée payante pour les autres. Il est hors de doute que le recours à la violence soit donc une justification utilitaire, c'est-à-dire la conviction qu'elle paie en permettant la défense des intérêts menacés du groupe contestataire. C'est dans ce contexte que l'utilisation intelligente de la violence est restée certainement une puissance structurante de l'espace politique transitionnel au CongoKinshasa. Elle est enlisée et loin d'être finie, vu le fonctionnement des institutions de la République sur fond de crise de confiance, de tensions, des désaccords sur les règles du jeu etc.

Tout compte fait, cette réflexion prend pour opératoire la définition proposée par Harold-Léonard Nieburg, à savoir, des actes de désorganisation, destruction, blessures, dont l'objet, le choix des cibles ou des victimes, les circonstances, l'exécution, et/ou les effets acquièrent une signification politique, c'est-à-dire tendent à modifier les comportements d'autrui dans une situation de marchandage qui a des conséquences sur le système social ${ }^{11}$. Les présentes lignes auront effectivement pour but de contribuer à cerner ce recours à la méthode violente et à pénétrer la signification politique que lui auraient conféré les entrepreneurs de la violence politique sous examen.

\section{LA VIOLENCE POLITIQUE SOUS L'AFDL ET LA PARTICIPATION POLITIQUE}

La violence politique menée sous le label de l'Alliance des Forces Démocratiques pour la Libération (AFDL) du Zaïre, sous le mot d'ordre de lutte contre l'accaparement de l'Etat par Mobutu et la partitocratie notamment, bénéficiât d'un crédit populaire sans mesure, au point que sa trajectoire fut de très courte durée. Commencée en 1996, la guerre fut son apogée le 17 mai 1997, avec la chute du régime contesté de Mobutu. Ce régime monopolistique, incrusté des malaises sociaux, des crises des légitimités et sclérose du pouvoir, n'avait pas survécu à la nouvelle conscience avivée des citoyens d'ouvrir le pays à la démocratie et à la gouvernance du développement. Cette crise, décorant l'apocalypse de Mobutu, avait donc sapé la légitimité des institutions étatiques sous son règne, incapables de remplir les fonctions sociales qui sont les siennes.

La tranche populaire, cacique victime de l'effondrement des systèmes éducatif, sanitaire, d'emploi, ... en vient à douter que toute légitimité sociale ne garantissait pas l'égalité des chances entre citoyens Zaïrois. Le recours à la violence fut, dans ce cas d'école, un moyen d'échapper aux malaises sociaux dont la société zaïroise fut le théâtre. A ces propos, affirme Jean William Lapierre, [...] le recours à la violence caractérise aussi les sociétés dans lesquelles un pouvoir politique abusif, oppressif, incapable de remplir sa fonction sociale a laissé, par impuissance ou par aveuglement, ou par solidarité avec «le désordre établi », se former peu à peu une situation révolutionnaire ${ }^{12}$. Cependant, l'existence de la violence, fondement du changement social dans ce cas

\footnotetext{
${ }^{7}$ T. Gurr cité par P. Braud, Op. cit., p.473.

${ }^{8}$ P. Braud, Op. cit., p.473.

${ }^{9}$ Idem, p.471.

${ }^{10}$ T. Gurr cité par P. Braud, Op. cit., pp.473-474.

${ }^{11}$ H.-L. Nieburg, Political violence. The behavioral process, New York, St Martin's Press, 1969, p.13.

12 J.-W. Lapierre « La violence dans les conflits sociaux», in J. Onimus (Dir.), La violence dans le monde actuel, Desclée de Brouwer, Centre d'études de la civilisation contemporaine, 1968, p.132.

DOI: $10.9790 / 0837-2205067582 \quad$ www.iosrjournals.org $\quad 77 \mid$ Page
}


précis, n'est pas donc l'expression de la disparition de l'Etat congolais, plutôt son dysfonctionnement, sa faiblesse à assumer ses fonctions sociales, particulièrement l'emploi, l'éducation, la santé, l'amélioration des qualités de vie des populations, des efforts dans la réduction de la pauvreté par la prise des politiques publiques avec incidence sur les activités économiques, la lutte pour le respect des droits humains et libertés politiques individuelles comme collectives, l'alphabétisation des jeunes, etc.

Ce cliché sombre de la crise politique au Zaïre, parodié des malaises sociaux, fut vecteur de l'entreprise de la politique violente sous l'AFDL, ovationnée par les peuples, hiérarques victimes de la dérive totalitaire du régime de Mobutu. Pour ce régime contesté, la gouvernance semblait se passer comme si la démocratie, avec toutes ses exigences, était, à l'exemple du valet noir, le pouilleux du jeu du mistigri, la carte qu'il faut inéluctablement échapper ou dont il faut, si on l'a attrapée, se libérer si possible pour le compétiteur à venir. Pourtant, elle implique une gestion d'austérité, plus normée et consciente ; une manière efficace et efficiente de gestion de la chose publique par des acteurs puisés dans la sphère étatique comme privée ainsi que de la société civile, dans l'intérêt premier d'améliorer les conditions d'existence de tous, par la stabilisation et la maîtrise de l'espace politique, économique et social. Et pour reprendre Guy Hermet, la gouvernance est le nom de l'après démocratie $^{13}$.

Cette violence avait conduit à l'instauration d'un nouveau régime le 17 mai 1997, avide d'une gouvernance démocratique et développeur. Et comme le souligne justement Maurice Duverger, toute contestation de l'ordre social existant est image et projet d'un ordre supérieur, plus authentique. Toute lutte porte en elle un rêve de paix, et constitue un effort pour l'incarner ${ }^{14}$. Les institutions politiques issues de cette violence politique furent mises en place par le Décret-loi constitutionnel nº03 du 27 mai 1997 relatif à l'organisation et à l'exercice du pouvoir au Congo-Kinshasa. Ce décret fut, pour des raisons de commodité démocratique, à deux reprises révisées. Premièrement, par le Décret-loi constitutionnel n074 du 25 mai 1998 et, deuxièmement, par celui $\mathrm{n}^{\circ} 096 / 2000$ du $1^{\mathrm{e}}$ juillet 2000.

Cependant, loin d'observer la démocratie et la gouvernance participative tant attendues, le nouveau régime (AFDL) fut prématurément acculé à la disparition par les mouvements rebelles RCD et MLC. Car, l'affirme T. Leary, les révolutions se sont toujours limitées à leurs dimensions extérieures : pour cette raison, elles n'ont fait que remplacer une dictature par une autre..., remplacer un groupe armé par un autre, qui devient aussi répressif que le précédent. En somme, un échange de gardiens de prison : la politique est une lutte pour savoir qui contrôlera les clefs de la prison... Pour briser ce cycle, la libération intérieure doit précéder la libération extérieure ${ }^{15}$. Ces mouvements RCD et MLC avaient, respectivement, mobilisé avec eux, des milliers des soldats rwandais et ougandais pour leur cause.

\section{LA VIOLENCE POLITIQUE SOUS LE RCD, UN ANTIDOTE A LA JEUNE DICTATURE ?}

A la genèse du mouvement rebelle Rassemblement Congolais pour la Démocratie (RCD), il était difficile, par les moyens légaux, d'investir le champ politique congolais, dont le levier de commande revenait de fait à M. Laurent-Désiré Kabila et son AFDL. Comme susmentionné, ce dernier venait de mettre en vigueur un Décret-loi constitutionnel $\mathrm{n}^{\circ} 003$ du 27 mai 1997 devant régenter les institutions politiques du pays. Ce cadre juridique avait conduit à la centralisation des pouvoirs exécutif et législatif entre les mains du président de la République, la suspension des activités des formations politiques sous l'ensemble du territoire national à l'exception de l'AFDL, la dissolution du parlement, ... Ce décret-loi n'avait pas prévu les procédures d'alternance au niveau de la magistrature suprême.

Pour Jean Otemikongo Mandefu, ce raisonnement donne à penser que le président Laurent-Désiré Kabila et son AFDL n'avait pas en tête l'idée de poursuivre le processus de démocratisation engagée sept ans avant (1990). Il s'agissait plutôt de remettre le chronomètre politique à zéro pour redémocratiser le pays pendant une nouvelle période de deux ans, sous l'autorité de l'AFDL, en vue de consolider les acquis de la libération ${ }^{16}$. L'unique voie possible à exploiter était de prendre exemple sur la jurisprudence Laurent-Désiré Kabila et son AFDL, c'est-à-dire investir la scène politique par la violence qui, de l'avis d'Andrée Nouschi, permet de

\footnotetext{
${ }^{13}$ G. Hermet, «La gouvernance serait-elle le nom de l'après démocratie? L'inlassable quête du pluralisme limité »,in G. Hermet, A. Kanzancigil et al. (Dir.), La gouvernance, un concept et ses applications, Paris, Karthala, 2005, p.17.

${ }^{14}$ M. Duverger, Sociologie politique, Paris, Presses Universitaires de France, 1968, p. 246.

${ }^{15}$ T. Leary cité par R.-G., Schwartzenberg, Sociologie politique, Paris, Montchrestien, 1998, p.355.

${ }^{16}$ Otemikongo Mandefu, «La transition démocratique à l'épreuve des faits en République Démocratique du Congo : examen critique de l'exercice du pouvoir pendant la période de transition », in Congo-Afrique, $\mathrm{n}^{\circ} 344$, pp 2006, pp.220-221. 
débloquer une situation dans laquelle la libre discussion a disparu, et la liberté de choix n'existe plus. Ainsi, la liberté perdue engendre la violence ${ }^{17}$.

Ce verrouillage des moyens de lutte politique de velours a été catalyseur d'une explosion de la violence, menée par le mouvement rebelle RCD depuis le 2 août 1998. Cet assaut frontal est savamment justifié par Maurice Duverger en trois possibilités : quand l'opposition n'a pas d'autre moyen d'action, quand elle est privée de toute autre forme d'expression, ou quand celle qu'on lui reconnaît est inefficace ${ }^{18}$. Ainsi, le mouvement avait eu à décrier la confiscation des tous les pouvoirs, la pratique du cumul de fonctions (exécutive et législative), la régionalisation de l'armée et la tribalisation des institutions politiques du pays, que Bertrand Badie appelle la culture communautaire du politique ${ }^{19}$, la remise en cause du système de gouvernance, etc.

Parmi les non-dits, il faut noter le détournement des objectifs de la coalition AFDL conformément au prétendu Accord de Lemera, coiffé par la décision du président Laurent-Désiré Kabila du 27 juillet 1998 demandant aux troupes militaires rwandaises et ougandaises notamment, de retourner dans leurs pays respectifs, alors confortablement installées au pouvoir et dans les mines au Congo-Kinshasa. Cette situation rejoint les propos de Philippe Braud en ce que « la violence revêt souvent le visage d'une protestation contre l'exclusion ou la marginalisation sur la scène institutionnelle ${ }^{20}$. Cette déconfiture du pays, occasionnée par sa désarticulation politique et sociale, fut révélatrice d'un dysfonctionnement du système politique, dont l'explication triangulaire est savamment présentée par Philippe Braud lorsqu'il soutient, parlant du système politique, soit qu'il ait affiché une indifférence foncière au problème dont la violence est le révélateur, soit qu'il ait considéré les solutions préconisées comme radicalement inacceptables pour sa survie, soit enfin, qu'il ait été incapable de percevoir correctement ou d'interpréter des signaux antérieurs de la nature non évidente ${ }^{21}$.

Ainsi, des jeux de rôle furent intervenus en vue du rétablissement de l'Etat dans son cadre fonctionnel normatif. Ce qui expliqua le lancement du Dialogue inter-congolais ayant accouché de l'Accord global et inclusif le 17 décembre 2002. Ce compromis établi, une des fonctions essentielles de la politique, n'a plus s'agit de régler le déroulement de la politique par la guerre, mais d'y mettre fin par un ajustement des intérêts en cause. Dans les régimes démocratiques, les institutions sont précisément aménagées à cet effet, leurs procédures ne servent pas seulement à exprimer les conflits politiques par des moyens non violents; elles sont aussi conçues pour y mettre fin, par la conclusion de compromis ${ }^{22}$. C'est dans cette logique, en effet, que la vie partisane du RCD n'a été possible qu'après l'abandon officiel de sa lutte guerrière. D'où le passage du RCD, de la lutte armée à la vie partisane, dénoterait de l'approche foucaultienne, selon laquelle, la politique, c'est la guerre continuée par d'autres moyens ${ }^{23}$. Parce quela guerre n'est pas à elle-même sa propre fin. Elle dépend de la politique et donc de l'Etat qui a la charge de conduire les affaires publiques. C'est la raison pour laquelle il importe de ne pas confondre les fins dans la guerre (Ziel) et de la guerre (Zweck): la fin dans la guerre, c'est la victoire militaire, alors que la fin de la guerre, c'est la paix, c'est-à-dire l'issue politique sur laquelle débouche tout conflit armé ${ }^{24}$

A tout bien prendre, l'accord du 17 décembre 2002 conditionna l'entrée en fonction du gouvernement de transition, formule $1+4$, le 30 juin 2003. Ainsi, les élites politiques rebelles furent parmi les nouveaux maîtres du pays dans les institutions politiques du gouvernement de salut public. Dans ce régime politique, en plus de la vice-présidence de la République en charge de la Commission politique, défense et sécurité, le RCD avait piloté 7 ministères sur un total de 36 soit $19 \%$ et 4 vices ministres sur un total de 36 soit $11 \%$. Dans la configuration du Parlement, le parti avait occupé 94 sièges à l'Assemblée national sur un total de 500 soit 18,8\% et 22 autres au Sénat sur un total de 120 sièges soit $18.8 \%$. Dans la gestion des Provinces, le parti avait géré 2 provinces sur un total de 11 soit $18 \%$. Ces rôles assumés dans les institutions politiques du pays leur avaient permis de se socialiser avec le corps social congolais et, par-dessus tout, se présenter aux élections organisées depuis 2006.

Par ailleurs, il importe de souligner que les intérêts restent au cœur des activités des groupes et les divisent parfois. La lutte politique du RCD n'avait pas dérogé à la fatalité segmentaire. Des dissidences l'ont traversées et l'histoire nous a instruit à cet effet. Parmi ces fragmentations les plus importantes, il y a le Rassemblement Congolais pour la Démocratie-Kisangani/Mouvement de Libération, RCD-KML en sigle et le

\footnotetext{
${ }^{17}$ A. Nouschi ; «La violence et le Tiers Monde », in J. Onemus. (Dir.), La violence dans le monde actuel, Desclée de Brouwer, éd. du Centre d'Etudes de la Civilisation contemporaine, 1968, p.183.

${ }^{18}$ M. Duverger, Op. cit, p. 249.

${ }^{19}$ B. Badie, «Communauté, individualisme et culture », in P. Birnbaum et J. Leca (Dir), Sur l'individualisme, théories et méthodes, Paris, Presses de la Fondation Nationale Science Politique, 1986, p.122.

${ }^{20}$ P. Braud, Op. cit., p.469.

${ }^{21} \mathrm{P}$. Braud, «La violence : repères et problèmes», Cultures\&Conflits, 09-10, printemps-été, [En ligne], mis en ligne le 13 mars 2006. URL http://conflits.revues.org/index406.html. Consulté le 4 septembre 2012.

${ }^{22}$ M. Duverger, Op. cit., p.303.

${ }^{23}$ M. Foucault, Il faut défendre la société, Paris, Seuil-Gallimard-EHESS, 1976, p.16.

${ }^{24} \mathrm{R}$. Aron, Penser la guerre, Clausewitz (2 tomes), Paris, Gallimard, 1976, p.170. 
Rassemblement Congolais pour la Démocratie/National, RCD-N en sigle, respectivement, renégat direct et indirect du RCD. Tous ces groupes se sont versés à la lutte acharnée du pouvoir politique à travers la revitalisation de la violence. Celle-ci leur a été payante chacun à son degré. Dans le gouvernement de transition, le RCD-KML et le RCD-N ont chacun occupé 2 ministères et 2 vices ministères. Dans le Parlement, ils ont respectivement occupé 15 et 5 sièges à l'Assemblée nationale et 4 et 2 sièges au Sénat. Dans la responsabilisation de provinces, ces mouvements ont dirigé chacun 1 Province.

\section{V.LA GUERRE DU MLC}

Paru comme une alternative congolaise à l'envahissement rwandais de la scène politique congolaise par l'entremise du RCD, le Mouvement de Libération du Congo (MLC) fut créé en novembre 1998 par Jean Pierre Bemba Gombo avec le soutien ougandais. Le mode opératoire du mouvement est la violence. Tout de même, il se veut nationaliste, et donc anti-rwandais. Par ces mots fédérateurs, le mouvement avait gagné la confiance d'une tranche populaire hostile à la présence rwandaise au Congo-Kinshasa sous l'AFDL comme sous le RCD.

La conjoncture sociale et politique était donc bien favorable à la lutte avec crédit populaire d'autant plus que Laurent-Désiré Kabila, après s'être confortablement installé sur le trône à Kinshasa, venait de marquer sa volte-face à ces mentors le 27 juillet 1998 en décidant du retour des troupes rwandaises et ougandaises notamment dans leurs pays respectifs, dont son régime est fantoche. Plus pessimistes, Tabin Lissendja Bahama et John Koko Lipambala opposent une fin de non-recevoir à la digestion de la présence rwandaise, en ces termes : «S'il y a un acte posé par le RCD que la population congolaise n'oubliera pas si vite, c'est d'avoir accepté et autorisé aux troupes étrangères de bafouer l'intégrité territoriale de la RDC. Dans le bilan négatif de l'action insurrectionnelle $\mathrm{du} \mathrm{RCD}$, il faut dire que beaucoup d'actes incriminés portaient le sceau des troupes d'agression ${ }^{25} »$.

En tout état de cause, l'usage de la violence est de facto un moyen d'accéder à l'existence politique en s'imposant comme interlocuteur aux différents acteurs du jeu institutionnel : les gouvernants bien sûr, mais aussi les autres parties et forces sociales contraints de se situer par rapport aux objectifs brillamment affichés ${ }^{26}$. Tout compte fait, la culture de participation politique par la violence a valu au MLC, après la normalisation politique, outre la vice-présidence de la République en charge de la Commission économique et financière, la gouvernance de 7 ministères sur un total de 36 soit $19 \%$ et 4 vice ministères sur un total de 36 soit $11 \%$. Au Parlement, le parti avait occupé 94 sièges sur un total de 500 soit 18,8\% et 22 sièges au Sénat sur un total de 120 soit $18,8 \%$. Concernant la gestion des provinces, il avait en charge la direction de la Province de Bandundu.

\section{VI.LE MÄ̈-MAÏ: UN PHENOMENE DE RESISTANCE POLITIQUE PAR LA VIOLENCE}

Le phénomène maï-maï est une tradition d'éloge à la violence dans une variabilité de raisons, de sens selon les zones de son expression, la temporalité et le contexte, dont l'explication dépasse la conjoncture immédiate de la guerre. Il fonde sa stratégie de lutte sur l'eau consacrée, le fétiche comme le souligne avec justesse Sophia Mappa: «En Afrique, les fétiches (dawa) sont des alliés imaginaires, en même temps, constituent des stratégies de lutte ${ }^{27}{ }$. Cette argumentation donne à penser que le mode opératoire de lutte de ce groupe repose, par essence, sur la violence fondée sur les règles traditionnelles de croyance fétichiste.

Au-delà de ses racines très anciennes au Congo-Kinshasa, aussi vielle que son émancipation politique, le maï-mä̈sme ${ }^{28}$ est réapparu avec une contagiosité incontrôlable au Nord-Kivu depuis 1996 comme une solution rwandaise à l'envahissement du territoire congolais par la résistance à la «soif de terre » des forces d'occupation. D'où, expliquent Franck van Acker et Koen Vlassenroot, l'intervention violente de jeunes Congolais qui montrent une disposition croissante à agir contre ce qu'ils perçoivent comme la cause de la crise

\footnotetext{
${ }^{25}$ T. Lissendja Bahama et J. Nkoko Lipambala, « La transformation du RCD en parti politique », in Revue de l'IRSA, $\mathrm{n}^{\circ} 12$, août 2006, p.24.

${ }^{26}$ P. Braud, Op. cit., p.469.

${ }^{27}$ S. Mappa, Pouvoir traditionnels et pouvoir d'Etat en Afrique. L'illusion universaliste, Paris, Karthala, 1998,

p.65.
${ }^{28}$ Le rapport du GRIP le présente comme une originalité congolaise, et date des années 1960. Les hommes de Mulele avaient de moyens militaires souvent rudimentaires, rudimentaires, insuffisants et de loin inefficaces pour mener une guerre aussi ambitieuse. Ils utilisaient pour leur grande majorité des armes blanches (machettes, couteaux, flèches, lances, etc.). Ils croyaient à des forces surnaturelles incarnées dans l'eau (d'où le mot « Maï» qui veut dire «l'eau» en swahili du Congo) et dans des amulettes et tatouages. Ils pensaient que cette eau «bénite » et ces amulettes avaient les vertus de vivifier et de rendre invulnérables les personnes initiées. Toutefois, cette invulnérabilité était assortie de plusieurs conditions et prescriptions qu'il ne fallait aucunement transgresser. Cf. Qui arme les maï-maï ? Enquête sur une situation originale, Rapport du GRIP, Bruxelles, 2004, pp.5-9.
} 
actuelle, c'est-à-dire ce qu'ils appellent l'occupation rwandaise de leur province ${ }^{29}$, en vue de la protection de la terre ancestrale, don béni des aïeux, dont la grandeur doit être assurée par le peuple, comme scandé dans l'hymne nationale « Débout Congolais ».

Soulignons, cependant, que le véritable problème de la réapparition du phénomène maï-mä̈ n'est pas tant constitué par l'existence de ceux qu'il convient d'appeler les « assoiffés des terres » (les forces d'occupation), mais de politiques publiques exclusives et sans mesure aux défis sociaux réels, car une politique mal pansée entraîne des désagréments dans la société et produit plus de problèmes que de solutions. Il s'agit là d'une expression de l'«Etat dormeur », pour dire un pouvoir dysfonctionnel à remplir sa mission de sécurité et de régulateur de la vie sociale et économique que se sont développés les réflexes d'auto-prise en charge populaire, spécifiquement dans le domaine de la protection physique et patrimoniale, et de la participation politique.

C'est dans ce contexte que le maï-maï, un des acteurs de la violence au Congo-Kinshasa, est analysé sous l'approche clausewitzienne, selon laquelle, « la guerre n'est pas seulement un acte politique, mais un véritable instrument politique, une poursuite des relations politiques, une réalisation de celles-ci par d'autres moyens ${ }^{30} \gg$. On le voit, cette approche met à son centre d'intérêt le rapport de force. «La guerre n'est qu'une partie des rapports politiques, et par conséquent nullement quelque chose d'indépendant ». «La guerre n'est rien d'autre que la continuation des relations politiques, avec l'appoint d'autres moyens ${ }^{31}$. Cette pensée de Carl von Clausewitz définissant le phénomène maï-maï, fait de la guerre un «instrument politique ${ }^{32}$ ». La guerre en serait, vraisemblablement, en effet, un outil tandis que la politique passerait pour le projet auquel cet outil est relativement destiné. Cet usage du concept instrument insinue l'existence d'une panoplie de modes de manifestation de la politique bien au-delà de la guerre, celle-ci n'en étant qu'une des modalités d'usage.

C'est pourquoi, toutes les vagues de guerre que le Congo-Kinshasa a connues depuis 1996, n'ont pas manqué de croiser les forces de résistance maï-maï, dans leur hétérogénéité, sur leur chemin. Ce faisant, le mouvement maï-maï était devenu, par la force de la violence, un des acteurs clé de la normalisation politique au Congo-Kinshasa, dont l'Accord global et inclusif en est la résistante. Dans les institutions gouvernementales entrées en fonction le 30 juillet 2003, le maï-mä̈ avait piloté 2 ministères sur un total de 36 soit 5,5\% et 2 vices ministères sur un total de 36 soit 5,5\%. Dans la configuration du Parlement, il avait occupé 10 sièges à l'Assemblée nationale sur un total de 500 et 4 sièges au Sénat sur un total de 120 soit respectivement $2 \%$ et $3 \%$. Dans la répartition des provinces, elle avait la charge de diriger la Province du Katanga.

\section{CONCLUSION}

Il existe une variabilité de formes de participation politique que l'on ne peut réduire au vote, bien que celui-ci soit la forme avivée dans les démocraties modernes où, malheureusement, recourir aux méthodes violentes, associées aux moyens pacifiques (barricade routière, occupation illégale de la voie publique, grève, sit-in ...), constitue un signe du danger dont ils ne peuvent s'épargner.

Cette acception donne à penser que la violence ${ }^{33}$ demeure un mode relativement expéditif de captation du pouvoir, un moyen court, un raccourci pour accéder à des positions d'autorité. Cependant, non seulement elle l'est, mais appelle à l'anti-constitutionnalité de l'action. Elle est un signal fort du danger que guette le fonctionnement d'un système politique en proie à la récurrence de cas de violence dans une infinité de manifestation et de raison, et consacre son affaiblissement aussi rédhibitoire que son incapacité régulatrice plus que responsive. C'est à n'en point douter la causalité de la revitalisation de la violence dans l'univers politique congolaise.

De plus, cet argumentaire prouve combien l'Etat congolais, laminé par des malaises sociaux, des contestations de légitimité et verrouillage des voies de lutte démocratique s'est exposé à l'explosion de la violence pour désavouer les institutions politiques incapables de gouverner le pays à sa grandeur et à ses innombrables richesses au profit du mieux-être du citoyen, et les substituer par un autre régime souhaité démocratique et dont la prescription est d'établir un système social juste et équilibré, où la distribution/redistribution des ressources collectives est fonction du mérite (proportionnalité entre les

\footnotetext{
${ }^{29} \mathrm{~F}$. Acker (van) et K. Vlassenroot, «Les «maï-maï» et les fonctions de la violence milicienne dans l'est du Congo », in Politique africaine, $\mathrm{n}^{\circ}$ 84-décembre 2001, p.104.

${ }^{30}$ C. Clausewitz (von) cité par C.-P. David et J.-J. Roche, Théories de la sécurité, Paris, Montchrestien, 2002, p.20.

${ }^{31}$ K. Clausewitz (von), De la guerre (1832), trad. D. Naville, Paris, Éditions de Minuit, 1955, p.703.

${ }^{32}$ Idem, p.43.

${ }^{33}$ Son antonyme la non-violence est un mode pacifique de conquête du pouvoir et nettement indiqué dans les systèmes à tradition démocratique. Il est relativement plus couteux et très complexe pour accéder au pouvoir et dure généralement longtemps. D’où, elle appelle à la persévérance, à la capacité persuasive, à la confiance en soi, à la mobilisation des ressources nécessaires en vue, non seulement d'être connu par le plus grand public, mais de devenir au mieux sa préférence lorsque vient le vote.
}

DOI: $10.9790 / 0837-2205067582 \quad$ www.iosrjournals.org $\quad 81 \mid$ Page


contributions et les gratifications des participants), de besoin ( découplage des contributions et des gratifications et répartition en fonction de l'utilité espérée) et du statut (répartition sur la base de l'identité sociale des membres, avec pour cas particulier important l'égalité) ${ }^{34}$, ingrédient aux flux des soutiens populaires au régime. Cependant, les attentes populaires quant à ce, sont restées déçues par le remplacement d'une dictature par une autre, dans un continuum d'oppressions aussi récusables que la crise démocratique de régime répressif précédent.

A l'inverse, nous voudrions affirmer deux facettes de la violence, qui ne sont d'ailleurs qu'un truisme au regard des clivages qui opposent les acteurs centraux du champ politique congolais. D'une part, les facteurs qui contribuent à rendre la violence moins justifiée ou moins efficace. Ce sont pour l'essentiel les soutiens dont peut se targuer le régime constitutionnel ${ }^{35}$. Si le régime de Mobutu était à la hauteur de ses responsabilités d'Etat, et le peuple reconnu détenteur de la souveraineté et placé au centre des actions gouvernementales du développement socioéconomique, la guerre de l'AFDL ne saurait être ovationnée et poussée à la victoire par le peuple avec l'ampleur des flux des soutiens populaires qu'elle a bien mérité.

D'autre part, lorsque les gouvernants disposent des moyens techniques appropriés pour maintenir l'ordre : forces de police nombreuses, loyales, et bien équipées, ils élèvent le seuil à partir duquel la violence se révèle efficace. Soit parce qu'ils tuent dans l'œuf tout processus de désordre, soit parce qu'ils en font payer chèrement le prix (répression sévère dans les dictatures, ...), soit surtout parce qu'ils utilisent des techniques évoluées permettant de canaliser l'agressivité destructrice ${ }^{36}$. C'est la raison pour laquelle le Congo-Kinshasa, avec toutes les illusions qui entourent cet ambitieux ouvrage, procède sans relâche, avec l'aide des partenaires, à la réforme des forces de sécurité et à leur équipement spécialisé. Les brouillards de la force du maintien de l'ordre, avec capacités requises de technicité et performance, peine à s'installer depuis 2002. Elle est encore à la traine des intérêts mesquins, ingrédient à ses dissidences et sa fragilité.

\section{BIBLIOGRAPHIE}

[1] Acker (van) F. et Vlassenroot K., «Les «maï-maï» et les fonctions de la violence milicienne dans l'est du Congo », in Politique africaine, $\mathrm{n}^{\circ}$ 84, décembre 2001, pp.103-116.

[2] Aron R., Penser la guerre, Clausewitz (2 tomes), Paris, Gallimard, 1976.

[3] Badie B., «Communauté, individualisme et culture », in Birnbaum P. et Leca J. (Dir), Sur l'individualisme, théories et méthodes, Paris, Presses de la Fondation Nationale Science Politique, 1986, pp.109-131.

[4] Boudon R., Besnard P. et al.,Dictionnaire de sociologie, Paris, Larousse, 1999

[5] Braud P., Sociologie politique, Paris, L.G.D.J, 2011.

[6] Braud P., « La violence : repères et problèmes», in Cultures\&Conflits, 09-10, printemps-été, [En ligne], mis en ligne le 13 mars 2006. URL http://conflits.revues.org/index406.html. Consulté le 4 septembre 2012.

[7] David C.-P. et RocheJ.-J., Théories de la sécurité, Paris, Montchrestien, 2002.

[8] Clausewitz (von) K., De la guerre (1832), trad. D. Naville, Paris, Éditions de Minuit, 1955.

[9] Diangitukwa F., Qu'est-ce que le pouvoir ? Paris, L'harmattan, 2004.

[10] Duverger M., Sociologie politique, Paris, Presses Universitaires de France, 1968.

[11] Foucault M., Il faut défendre la société, Paris, Seuil-Gallimard-EHESS, 1976.

[12] GRIP, Qui arme les maï-maï? Enquête sur une situation originale, Rapport du GRIP, Bruxelles, 2004.

[13] Hermet G., «La gouvernance serait-elle le nom de l'après démocratie? L'inlassable quête du pluralisme limité »,in Hermet G., Kanzancigil A. et al. (Dir.), La gouvernance, un concept et ses applications, Paris, Karthala, 2005, pp.1747.

[14] Lapierre J.-W., «La violence dans les conflits sociaux», in Jean Onimus (Dir), La violence dans le monde actuel, Desclée de Brouwer, Centre d'études de la civilisation contemporaine, 1968, pp.129-158.

[15] LissendjaBahama T.et NkokoLipambala J., «La transformation du RCD en parti politique », in Revue de l'IRSA, $\mathrm{n}^{\circ} 12$, août 2006, pp.14-27.

[16] Mappa S., Pouvoir traditionnels et pouvoir d'Etat en Afrique. L'illusion universaliste, Paris, Karthala, 1998.

[17] Nieburg H.-L., Political violence. The behavioral process, New York, St Martin's Press, 1969.

[18] Nouschi A., «La violence et le Tiers monde» in Onimus J., (Dir.), La violence dans le monde actuel, Desclée de Brouwer, Centre d'études de la civilisation contemporaine, 1968, pp.183-198.

[19] Otemikongo Mandefu J., «La transition démocratique à l'épreuve des faits en République Démocratique du Congo : examen critique de l'exercice du pouvoir pendant la période de transition », in Congo-Afrique, n³44, 2006, pp.220241.

[20] Schwartzenberg R.-G., Sociologie politique, Paris, Montchrestien, 1998.

\footnotetext{
${ }^{34}$ R. Boudon, P. Besnard et al., Dictionnaire de sociologie, Paris, Larousse, 1999, p.130.

${ }^{35}$ P. Braud, Op. cit., p.474.

${ }^{36}$ Idem
} 